\title{
e-Interview
}

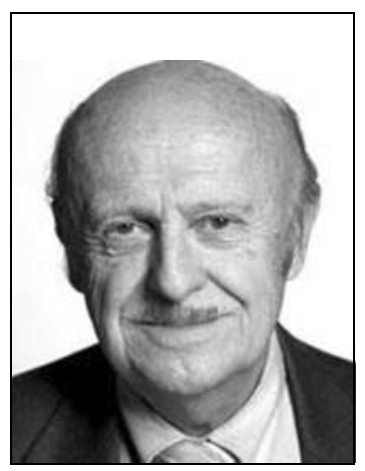

\section{James J. Strain}

James Strain is Professor and Director, Emeritus, of the Consultation-Liaison Behavioral Medicine service, Mount Sinai School of Medicine, New York, USA. He trained at the Case Western Reserve School of Medicine, Cleveland, Ohio, and the New York Psychoanalytic Institute. His special interests include electronic medical records, development of teaching models for mental health training of nonpsychiatric physicians, and depression as a systemic illness.

If you were not a psychiatrist, what would you do?

I would like to be an architect, creating orderly and imaginative spaces for persons to live in; like freeing up spaces in the mind by effective psychotherapy.

What has been the greatest impact of your profession on you personally? How much patients can benefit from the new interventions that are available in psychiatry.

What are your interests outside of work? Travel (194 countries visited), skiing, scuba diving, opera, dancing, swimming, collecting African art, reading about how persons have made their lives successful.

Would you go into psychiatry again, if you had your professional life to do over? Yes.

Who was your most influential trainer, and why?

Dr George Bidder (neurobiological systems), Dr Charles Brenner (psychoanalytic thinking) and Dr Jay Strain (an electronic medical record). These three quite disperate approaches to psychiatric modelling and thinking have given a breadth to my views of human behaviour and studying it.

What job gave you the most useful training experience?
Administering a consultation/liaison psychosomatic service where one had to always keep in mind the effects of body on mind and mind on body - psychosomatic and somatopsychic phenomena.

\section{Which publication has influenced you} most?

The American Journal of Psychiatry and its constant review of new findings, thoughts and ideas.

How has the political environment influenced your work?

The unavailability of mental healthcare for so many in such a wealthy country. The lack of parity for mental healthcare and physical care. Health plans that inadequately provide for mental healthcare.

\section{What part of your work gives you the} most satisfaction?

Teaching, patient care, and research trying to understand those with physical and mental comorbidity: a most neglected patient cohort both in medicine and psychiatry.

\section{What do you least enjoy?}

Trying to find a psychiatrist who will take care of a patient with our aberrant payment rates and schedules. Often patients on our government healthcare plans which do not pay a sufficient physician's fee means that patients go untreated or are undertreated.

What is the most promising opportunity facing the profession?

The development of neurobiological models that can serve as templates for the development of new drugs, early identification of potential illnesses (DSM-IV has no biological parameters to enhance diagnoses), and the ability to offer interventions that can be provided in primary care settings.

\section{What is the greatest threat?}

The somatic domain of medicine will co-opt resources badly needed for mental healthcare. The insurance companies absorption of $25-30 \%$ of healthcare dollars for administration and payment to shareholders, which should be directed towards patient care, research and teaching.

\section{What single change would substantially} improve quality of care?

A single-payer system; a governmentsponsored healthcare programme with parity for mental and physical healthcare needs.

What conflict of interest do you encounter most often?

Medicine's lack of interest in mental health issues of their patients, and psychiatry's reluctance to become more medically aware of psychiatric patients' needs.
What is the role of the psychiatrist in countries emerging from conflict? Dealing with the ravage of barbarous treatment of developing populations by their fellow man (e.g. Rwanda, Darfur, Bosnia, Sierra Leone). Application of mental health techniques to attenuate trauma, post-traumatic stress disorder, and the massive dislocation of families.

What is the most important advice you could offer to a new trainee?

Get trained in as many dimensions of the most interesting field of psychiatry as you can. Pick an area of interest and develop your skills to promote the translation of basic knowledge in the clinical setting.

Work at the cutting edge of an area you are interested in to make a meaningful contribution to the field.

What are the main ethical problems that psychiatrists will face in the future? Confidentiality with the new electronic medical record; respecting autonomy and the capacity of the patient for clinical decision making; distribution of scarce resources - justice; acknowledging patient's choice; insuring that research findings are truthful and valid; and significant disclosures of potential conflicts of interest.

Do you think psychiatry is brainless or mindless?

Neither. It has a most important brain and neurobiological basis, and it has a unique and powerful understanding of the mind. It is a most important discipline which encompasses both domains.

What single area of psychiatric practice is most in need of development?

Child psychiatry, psychopharmacological understanding, in particular drug-drug interactions, and translation of psychiatric information throughout the medical disciplines.

What single area of psychiatric research should be given priority?

Neurobiology to understand the mechanisms underlying schizophrenia and other psychoses, Alzheimer's disease and autism.

How would you like to be remembered? As having made a significant contribution to understanding the effects of the mind on the body and vice versa. I think depression is a systemic disease - affecting many body processes (e.g. cardiovascular, diabetes, cancer, HIV, and many others to be discovered). Depression is not just a mental disorder and must become a part of training of all disciplines of physicians.

Dominic Fannon

doi: 10.1192/pb.bp.109.028704 